\title{
Suicídio e lesões autoprovocadas: análise do perfil epidemiológico e prevalência dos casos no Brasil entre 1996 e 2019
}

\author{
Suicide and self-harm: analysis of the epidemiological profile and prevalence of cases in \\ Brazil between 1996 and 2019
}
Suicidio y auto infracción: análisis del perfil epidemiológico y prevalência de casos en Brasil entre 1996 y 2019

Beatriz Targino Araújo da Paixão ${ }^{1 *}$, Daniele Aparecida dos Santos², lane Camila Costa Silva ${ }^{3}$, Monique Mayara Morais ${ }^{4}$, Monique Camargo ${ }^{4}$, Marcela Wanderley Gianini ${ }^{5}$, Regina Lígia Gomes Ferreira $^{5}$, Roberto Oliveira Miaki ${ }^{6}$, Vitória Maria Moras Vicentino ${ }^{5}$, Bruno Augusto Lopes ${ }^{7}$.

\section{RESUMO}

Objetivo: Analisar a prevalência da violência autoprovocada e suicídio no Brasil, analisando causas, manejo, prevenção e cuidados pós-incidente. Métodos: Estudo transversal, descritivo, através de dados epidemiológicos, associado à revisão narrativa. As informações foram obtidas no DATASUS, nas subseções Sistema de Informação de Agravos de Notificação (SINAN) e Sistema de Informações sobre Mortalidade (SIM). Resultados: Nos casos de violência autoprovocada, há predominância do sexo feminino $(68,21 \%)$ e população de $20-29$ anos (23,45\%). A população masculina consta com $78,95 \%$ de óbitos. O maior número de óbitos foi entre $20-29$ anos (22,40\%) e metade eram brancos (50\%). Discussão: O aumento dos números de autoextermínio dentre jovens foi relacionado à situação socioeconômica. O sexo masculino utiliza instrumentos e armas mais irreversíveis, resultando em taxas de óbitos elevadas. Em casos de pacientes com tentativa de suicídio, as equipes de saúde devem ser notificadas quanto o risco de suicídio e a atenção tem que ser redobrada em trocas de turnos. Conclusão: O sexo masculino apresenta maior taxa de mortalidade, a raça carece mais estudos que reflitam sobre a realidade brasileira. É essencial assistência adequada com capacitação dos profissionais de maneira sistemática além de preenchimento completo das fichas de óbito.

Palavras-chave: Suicídio, Tentativa de suicídio, Prevalência, Epidemiologia.

\footnotetext{
ABSTRACT

Objective: To analyze the prevalence of self-inflicted violence and suicide in Brazil, tracing causes, management, prevention and post-incident care to avoid recurrences. Methods: Cross-sectional, descriptive study, using epidemiological data, associated with narrative review. The information was obtained from DATASUS, in the subsections Information System for Notifiable Diseases (SINAN) and Information System on Mortality (SIM). Results: In cases of self-inflicted violence, there is predominance of females (68.21\%) and the population aged $20-29$ (23.45\%). The male population accounts for $78.95 \%$ of deaths. The highest number of deaths was between $20-29$ years (22.40\%) and half were white (50\%). Discussion: The increase in the

${ }^{1}$ Universidade Católica de Brasília (UCB), Brasília - DF. *E-mail: btapaixao@gmail.com

2 Faculdade de Medicina de Valença (UNIFAA), Valença - RJ.

${ }^{3}$ Faculdade Unyleya (UNYLEYA), Brasília - DF.

4 Universidade Anhembi Morumbi (UAM), São Paulo - SP.

5 Universidade de Vassouras (UV), Vassouras - RJ.

${ }^{6}$ Pontifícia Universidade Católica do Paraná (PUCPR), Curitiba - PR.

7 Hospital Municipal Miguel Couto, Rio de Janeiro - RJ.
} 
number of self-extermination among young people was related to the socioeconomic situation. Males use more irreversible instruments and weapons, resulting in higher death rates. In patients with a suicide attempt, the health team needs to be notified of the risk of suicide and attention has to be increased during periods of shift changes. Conclusion: Males have a higher mortality rate, the race variable needs more studies about the Brazilian reality. Adequate care is essential with systematic training of health professionals, in addition to the complete completion of death forms.

Keywords: Suicide, Suicide attempted, Prevalence, Epidemiology.

\section{RESUMEN}

Objetivo: Analizar prevalencia de violencia autoinfligida y suicidio en Brasil, rastreando causas, manejo, prevención y atención post-incidente. Métodos: Estudio descriptivo, transversal, utilizando datos epidemiológicos, asociado a revisión narrativa. Información se obtuvo de DATASUS, en las subsecciones Sistema de Información de Enfermedades Notificables (SINAN) y Sistema de Información de Mortalidad (SIM). Resultados: En casos de violencia autoinfligida, predomina el sexo femenino $(68,21 \%)$ y 20 a 29 años $(23,45 \%)$. Población masculina representa $78,95 \%$ de las muertes. Mayor número de defunciones se produjo entre 20 y 29 años (22,40\%) y la mitad fueron de raza blanca (50\%). Discusión: Incremento en el número de auto-exterminio entre jóvenes se relacionó con la situación socioeconómica. Hombres usan más instrumentos irreversibles y armas, lo que resulta en tasas de mortalidad más altas. En pacientes con intento de suicidio, el equipo de salud debe ser notificado del riesgo y debe aumentarse la atención durante el cambio de turno. Conclusión: Hombres tienen una tasa de mortalidad más alta, la variable raza necesita más estudios que reflejen la realidad brasileña. Atención adecuada es fundamental, con formación sistemática de los profesionales de la salud, además de la cumplimentación de los formularios de defunción.

Palabras clave: Suicidio, Intento de suicidio, Prevalencia, Epidemiología.

\section{INTRODUÇÃO}

A violência autoprovocada compreende ações propositais cometidas pelo próprio indivíduo, e que resultam em injúria corporal, podendo envolver ideação suicida (WITT K, et al., 2018). No âmbito das autolesões sem intenção suicida (ASIS), a mais comum é a autoflagelação (ROBINSON K, et al., 2021; MINISTÉRIO DA SAÚDE, 2016). Já naquelas com intenção de óbito, tem-se o comportamento suicida, cujo caráter é gradativo, com início na ideação, seguindo para o planejamento do ato, a tentativa e eventual consumação (CANOMONTALBÁN I e QUEVEDO-BLASCO R, 2018).

O espectro suicida é um transtorno que resulta de uma abstrusa dinâmica multifatorial, envolvendo aspectos genéticos e ambientais (OMS, 2019). Existem muitos estudos sobre teorias e fatores de risco para comportamentos suicidas, porém, ainda há carência de evidências sobre elas na literatura (FRANKLIN JC, et al., 2016). Em relação às ASIS, podem ser entendidas como provenientes de repercussões das funções interpessoais e intrapessoais. Dentre elas, a última é a mais frequentemente associada e diz respeito à capacidade regulatória da emoção e à autopunição do indivíduo (TAYLOR PJ, et al., 2017).

Não obstante, aspectos como presença de doenças psiquiátricas e história de traumas são frequentemente associados tanto às autoagressões e suas recidivas, quanto ao espectro suicida (MAIS G, et al., 2020). Ademais, há indícios de que indivíduos com histórico de autoflagelação sejam mais propensos a tentar suicídio (ROBINSON K, at al., 2021; BUTTER S, et al., 2018; MARS B, et al., 2019).

Estes transtornos têm grande impacto em morbimortalidade e qualidade de vida na população (MINISTÉRIO DA SAÚDE, 2016). Segundo a Organização Mundial da Saúde (OMS), os casos de suicídio constituem a segunda principal causa de morte entre pessoas de 15 a 29 anos e são mais prevalentes em países em desenvolvimento. Cerca de 800.000 casos são registrados anualmente e a maioria é cometida por homens, enquanto as tentativas de suicídio são predominantemente realizadas por mulheres (BACHMANN S, 2018). 
Nesse sentido, notificações adequadas desses incidentes são primordiais para caracterizar essa população e traçar estratégias de prevenção e manejo. A educação de profissionais e estudantes da área da saúde, o seguimento de pacientes pós-alta e a restrição de acesso a meios letais, por exemplo, são possíveis condutas para a prevenção do suicídio em escala (MANN JJ, et al., 2021).

O presente estudo objetiva analisar a prevalência e perfil epidemiológico da violência autoprovocada e suicídio no Brasil, aprofundando suas causas, manejo, prevenção e cuidados pós-incidente.

\section{MÉTODOS}

Trata-se de um estudo observacional transversal, descritivo, através do levantamento de dados epidemiológicos, associado a uma revisão narrativa da literatura. As informações epidemiológicas e de morbidade, bem como as de estatísticas vitais e de mortalidade foram obtidas no Departamento de Informática do Sistema Único de Saúde (DATASUS), nas subseções do Sistema de Informação de Agravos de Notificação (SINAN) e do Sistema de Informações sobre Mortalidade (SIM) durante o mês de junho e julho de 2021.

O presente trabalho utilizou dados disponíveis para acesso público na plataforma DATASUS do Ministério da Saúde, assim sendo, esta pesquisa não necessitou de aprovação de Comitê de Ética em Pesquisa com seres humanos conforme a Resolução número 510 do Conselho Nacional de Saúde, de 7 de abril de 2016, artigo 1, inciso III, que isenta pesquisa que utilize informações de domínio público em Ciências Humanas e Sociais de registro no Comitê de Ética em Pesquisa da Comissão Nacional de Ética em Pesquisa - Sistema CEP/CONEP.

A coleta de dados no SINAN foi direcionada de acordo com os critérios de inclusão para os registros de notificações de lesões autoprovocadas, no período de janeiro de 2009 a dezembro 2019, segundo as variáveis sexo, faixa etária, cor/raça, escolaridade, ano e região de notificação, evolução do caso e reincidência. Já no SIM, foram incluídos os casos de óbitos confirmados relacionados a causas abrangidas pelo grupo da Classificação Estatística Internacional de Doenças (CID 10) e Problemas Relacionados com a Saúde (lesões autoprovocadas voluntariamente) e pelo grupo CID 10 X60 - X84 (lesões autoprovocadas intencionalmente) contando com a obtenção dos números absolutos discriminados segundo cada categoria do CID10, no período de 1996 a 2019.

Os critérios de exclusão dessa pesquisa foram: dados anteriores a janeiro de 1996 no SIM e anteriores a janeiro de 2009 no SINAN, notificações fora das categorias de lesões autoprovocadas, lesões autoprovocadas voluntariamente e lesões autoprovocadas intencionalmente.

Foi realizada uma revisão narrativa da literatura com base em 33 artigos selecionados pelos critérios de inclusão entre o período de 2016 e 2021, com uso dos descritores "suicídio", "tentativa de suicídio", "prevalência" e "epidemiologia". As bases de dados utilizadas foram PubMed, Latindex, Scielo, Directory of Open Access Journals (DOAJ), Lilacs e Biblioteca Virtual de Saúde (BVS), nos idiomas português e inglês, pesquisados durante o mês de junho, julho e agosto de 2021. Os critérios de exclusão foram: artigos publicados em anos anteriores a 2016, e que não contemplavam o tema.

\section{RESULTADOS}

\section{Sistema de Informação de Agravos de Notificação (SINAN)}

Através de dados epidemiológicos, obtidos pelo SINAN, foram analisadas as variáveis "sexo", "faixa etária", "cor/raça", "escolaridade", "ano de notificação", "região de notificação", "evolução do caso" e "violência de repetição", conforme as notificações realizadas no período de janeiro de 2009 a dezembro de 2019.

\section{Notificações}

Foram notificados 471.291 casos de lesão autoprovocada, sendo 2019 o ano mais expressivo, totalizando $26,87 \%$ dos casos. Em oposição, o ano de 2009 apresentou os menores números, com 0,83\% das notificações. 
Em relação ao aspecto geográfico, a região Sudeste destaca-se pelo número de casos $(48,31 \%)$, representando quase a metade de todas as notificações do país. Em oposição, encontra-se a região Norte, que apresenta os menores números de notificações (3,99\%), enquanto as regiões Sul, Nordeste e CentroOeste aparecem em posições intermediárias, respectivamente.

Por meio do exame das variáveis demográficas, o sexo feminino predomina $(68,21 \%)$, sendo a relação mulher/homem de aproximadamente 2,14:1. Sobre a faixa etária, destaca-se o intervalo entre 20-29 anos de idade, representando $23,45 \%$ do total. Sobre as notificações por cor/raça, a cor branca corresponde a aproximadamente $48,77 \%$ dos registros, enquanto somente $0,59 \%$ das notificações provêm de ocorrências entre as populações indígenas (Gráfico 1).

Quanto à escolaridade, casos ignorados ou em branco predominam $(37,72 \%)$, demonstrando que tal informação não foi incluída no documento de notificação compulsória. No extremo oposto, os pacientes analfabetos representam $0,75 \%$ do total de casos. O segundo maior número de notificações corresponde à faixa de "ensino médio completo", totalizando $14,57 \%$ dos casos (Figura 1).

No que concerne à reincidência dos casos, foi notificada repetição das lesões autoprovocadas em 35,06\% das vezes, sendo que em $41,18 \%$ dos casos não houve reincidência e em $0,55 \%$ das notificações, a recorrência foi registrada como "em branco". Da mesma forma, as notificações de evolução do caso foram deixadas "em branco" em $80,51 \%$ dos registros, ao passo que em $0,07 \%$ deles foi declarado "óbito por outras causas". Em 16,42\% das vezes os pacientes receberam alta.

Gráfico 1 - Notificações de Lesões Autoprovocadas pelo SINAN demonstrando o perfil epidemiológico mais prevalente

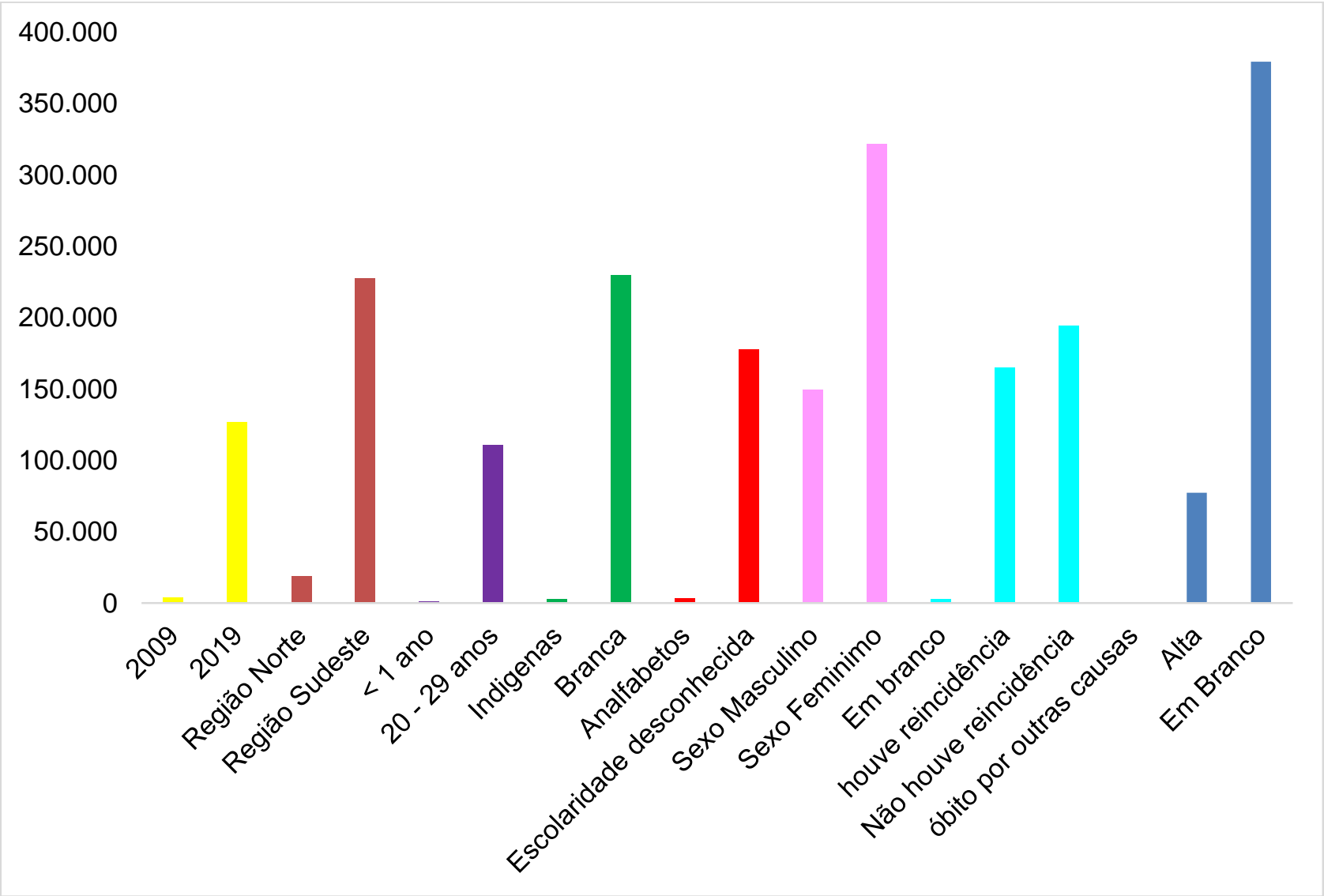

Fonte: Paixão BTA, et al., 2021. 
Figura 1 - Perfil epidemiológico do paciente mais afetado.

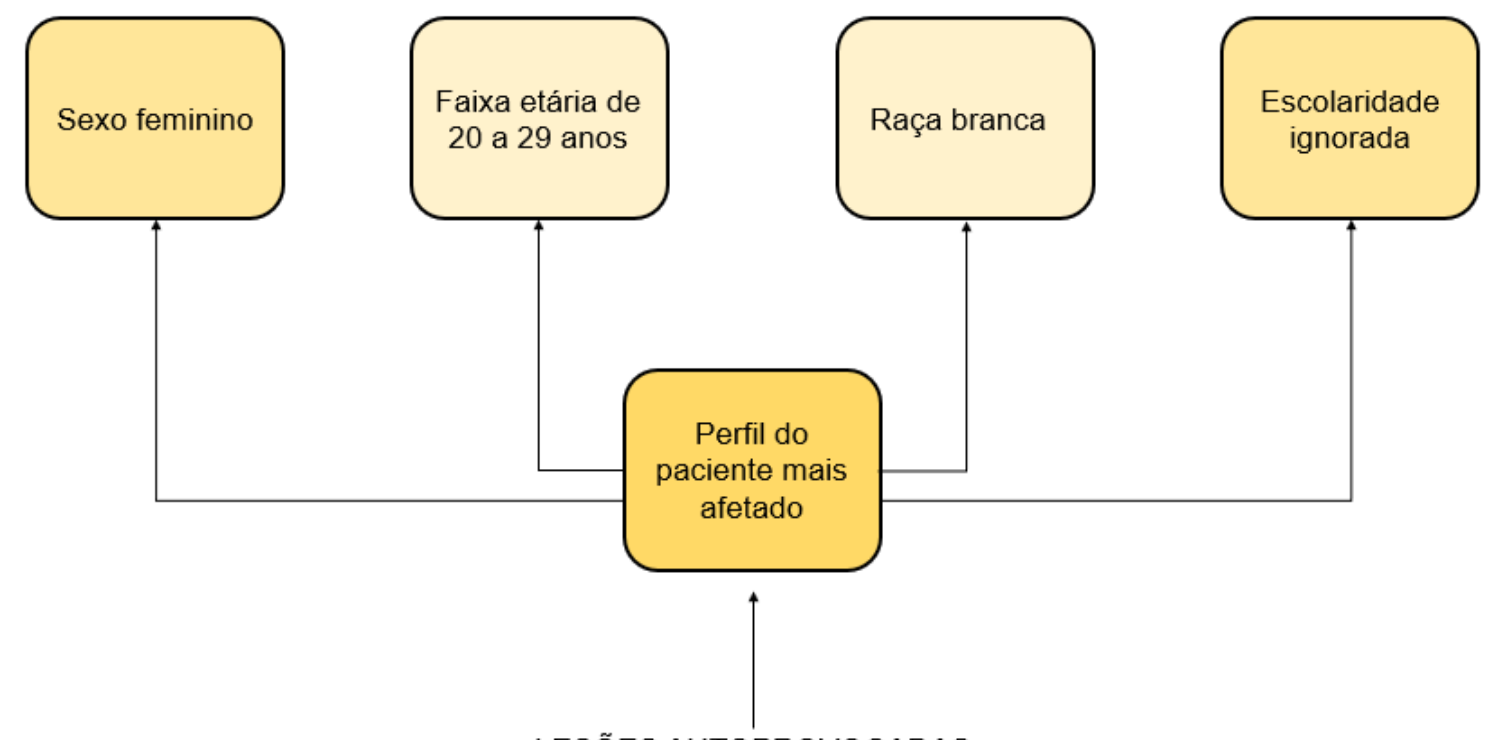

LESÕES AUTOPROVOCADAS

Fonte: Paixão BTA, et al., 2021.

\section{Sistema de Informações sobre Mortalidade (SIM)}

Os registros de óbitos foram obtidos no SIM através das seguintes variáveis: "região", "ano do óbito", "faixa etária", "sexo", "cor/raça", "escolaridade", "estado civil" e "local de ocorrência" entre 1996 a 2019.

\section{Óbitos}

O maior número de óbitos foi registrado em 2019 ( $6 \%$ do total de anos avaliados), enquanto apenas 3,04\% foram em 1996. Estes extremos revelam, ainda, um comportamento gradativo de aumento dos óbitos por ano, com exceção dos anos de 1999 e 2002. Entre 1996 e 2019, foram registradas 222.232 lesões autoprovocadas intencionalmente nas cinco regiões brasileiras. A região Sudeste se destacou com o maior número de casos, representando $38,20 \%$ da totalidade. Na sequência, identificou-se a Região Sul $(24,76 \%)$, Nordeste $(21,56 \%)$, Centro-Oeste $(9,01 \%)$. Em último lugar, encontra-se a região Norte, com 6,40\% dos suicídios.

A população masculina atingiu um índice de $78,95 \%$ dos óbitos, ficando a parcela feminina com $21,03 \% \mathrm{e}$ $0,02 \%$ são de sexo ignorado. A faixa etária com maior número de óbitos foi entre 20 e 29 anos, totalizando $22,40 \%$ do total avaliado. Entre 10 e 14 anos e 15 e 19 anos, a letalidade sobe de 1,27\% para 7,24\%. Ademais, quando reunidos os períodos etários de 20-29, 30-39 e 40-49 anos, observa-se $62,04 \%$ dos casos de lesão autoprovocada intencionalmente que resultaram em morte.

Ao avaliar a cor/raça dos indivíduos, metade dos que vieram a óbito eram brancos (50\%). Aqueles classificados como pardos e pretos representaram $34,17 \%$ e $4,96 \%$ do total, respectivamente. Em contraste, a população indígena $(0,89 \%)$ e os identificados como amarelos $(0,54 \%)$ apresentaram as menores taxas de mortes por lesões autoprovocadas. Da relação de óbitos registrados no período, a maior parte correspondia a pessoas solteiras $(49,10 \%)$ ou casadas $(30,47 \%)$. O restante dos dados se subdividiu nas categorias "ignorado" (7,27\%), "separado judicialmente" (5,89\%), "viúvo" (4,06\%), e "outro" (3,25\%).

A especificação da escolaridade foi ignorada em grande parte do levantamento, não sendo informada em $36,65 \%$ das perdas. Observou-se um pico no número de mortes naqueles que tinham entre 4 a 7 anos de estudo, gerando uma taxa de $20,28 \%$ dos falecimentos. Apesar disso, vale à pena destacar que outras faixas de escolaridade também alcançaram cifras importantes, sendo que aqueles que tinham de 8 a 11 anos de estudo somaram $16,31 \%$ dos óbitos, e os indivíduos que tinham entre 1 e 3 anos de estudo contabilizaram $13,30 \%$ dos suicídios. 
No que tange a causa mortis de suicídio, 60,60\% foram por enforcamento, estrangulamento ou sufocação, destacando-se por ser essa a categoria dos meios mais frequentemente empregados. Logo, está o uso de armas de fogo, correspondendo a $12,50 \%$ do total de casos. Em posições intermediárias figuram a utilização de pesticidas e outros tipos de produtos químicos $(8,82 \%)$, muitos deles descritos como "não especificados", e a autoadministração de drogas (4,24\%), incluindo medicações, substâncias biologicamente ativas, além da intoxicação voluntária por ingestão de álcool. Quanto ao local de ocorrência, 57,53\% aconteceram em domicílio, 19,91\% sucederam em estabelecimento de saúde. Cerca de 15,11\% foram em locais não especificados (não englobados nas demais categorias), 6,33\% em via pública e em 1,13\% a informação não foi fornecida (Gráfico 2 e Figura 2).

Tabela 1 - Mecanismos de lesões autoprovocadas/suicídio de acordo com CID-10.

\begin{tabular}{lc}
\hline Mecanismos de suicídio pela categoria CID10 & Número de óbitos \\
\hline Auto-intoxicação por e exposição, intencional, a/por: & \\
\hline Analgésicos, antipiréticos e anti-reumáticos, não-opiáceos & 190 \\
Drogas anticonvulsivantes (antiepilépticos) $\quad$ sedativos, & hipnóticos, \\
antiparkinsonianos e psicotrópicos não classificados em outra parte & 3.272 \\
Narcóticos e psicodislépticos (alucinógenos) não classificados em outra parte & 821 \\
Outras substâncias farmacológicas de ação sobre o sistema nervoso autônomo & 205 \\
Outras drogas, medicamentos e substâncias biológicas e às não especificadas & 4.245 \\
Álcool & 700 \\
Solventes orgânicos, hidrocarbonetos halogenados e seus vapores & 244 \\
Outros gases e vapores & 459 \\
Pesticidas & 12.464 \\
Outros produtos químicos e substâncias nocivas não especificadas & 7.143 \\
\hline
\end{tabular}

\section{Lesão autoprovocada intencionalmente por/pela:}

Enforcamento, estrangulamento e sufocação

134.677

Afogamento e submersão

Disparo de arma de fogo de mão

Disparo de espingarda, carabina, ou arma de fogo de maior calibre

Disparo de outra arma de fogo e de arma de fogo não especificada

20.292

Dispositivos explosivos

Fumaça, pelo fogo e por chamas

4.015

Vapor de água, gases ou objetos quentes

Objeto cortante ou penetrante

Objeto contundente

Precipitação de um lugar elevado

7.488

Precipitação ou permanência diante de um objeto em movimento

Fonte: Paixão BTA, et al., 2021; dados extraídos do Sistema de Informações sobre Mortalidade (SIM) da plataforma DATASUS do Ministério da Saúde. 
Gráfico 2 - Óbitos pelo SIM demonstrando o perfil epidemiológico mais prevalente.

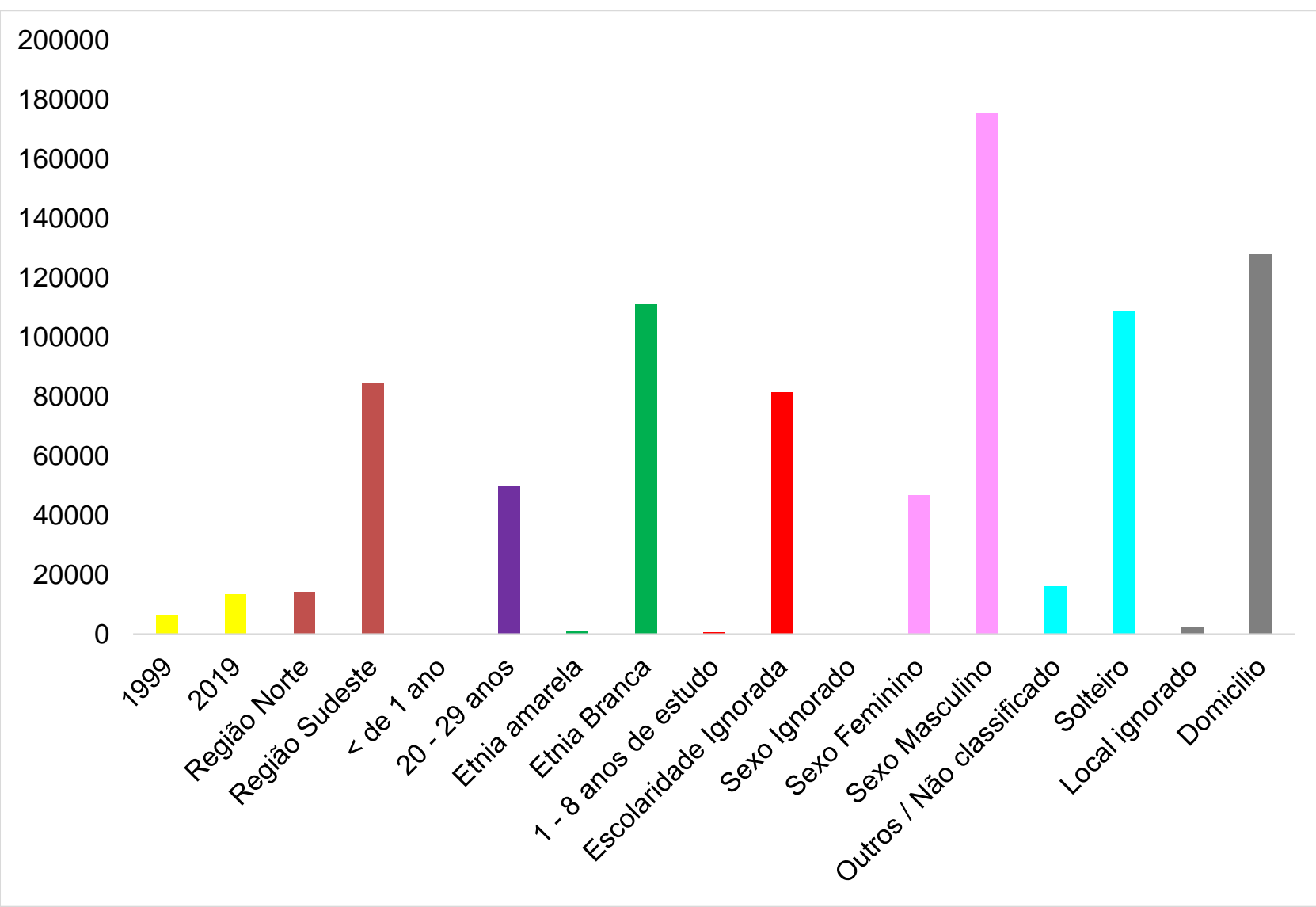

Fonte: Paixão BTA, et al., 2021.

Figura 2 - Perfil epidemiológico dos pacientes acometidos.

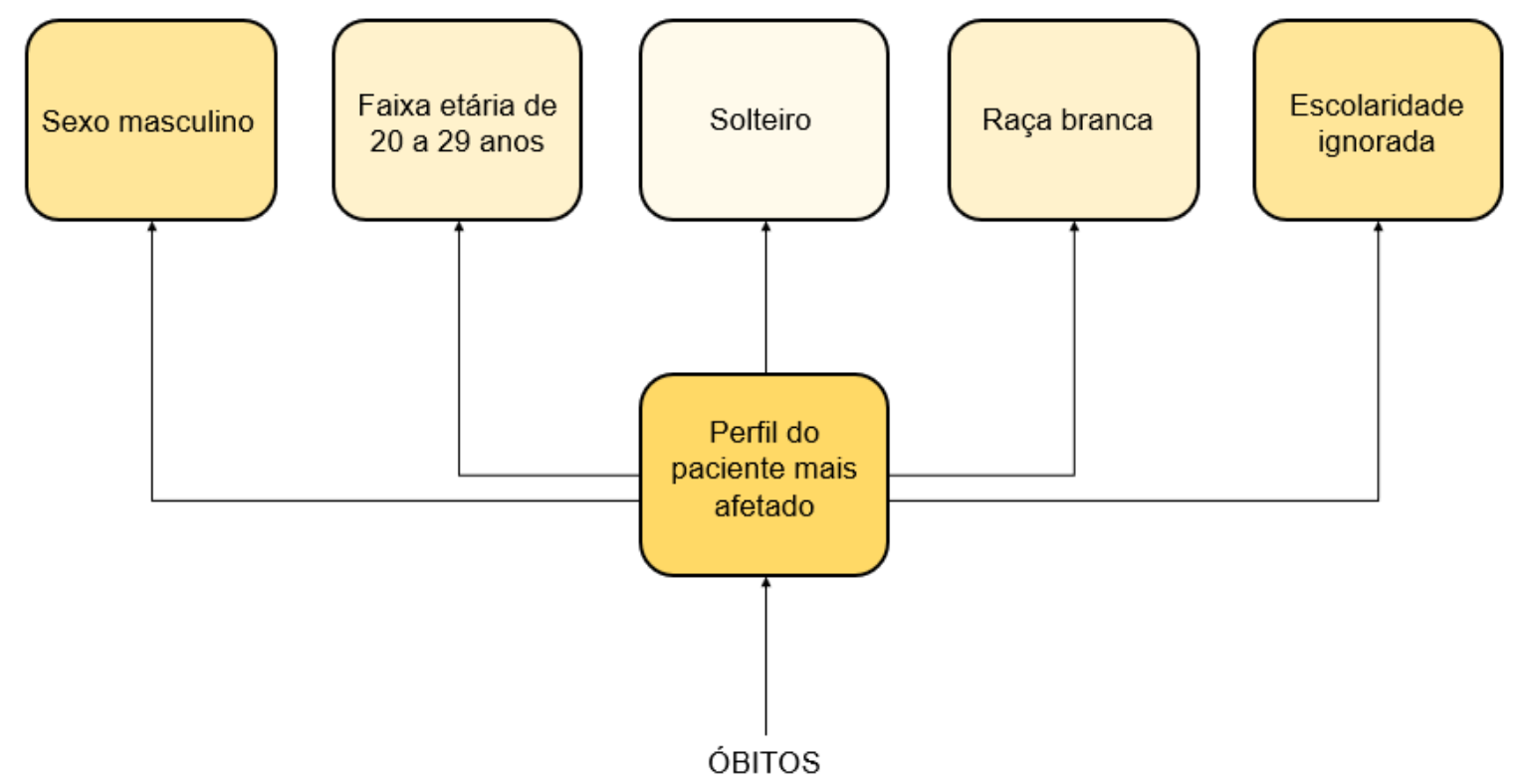

Fonte: Paixão BTA, et al., 2021. 


\section{DISCUSSÃO}

A lesão autoprovocada é a violência que o indivíduo provoca a si mesmo, sendo classificada como comportamento suicida ou autoagressão (WITT K, et al., 2018). O comportamento suicida é identificado quando uma pessoa lesa a si mesma, independentemente do grau de intenção letal ou do motivo real do ato. Nele engloba-se pensamentos, planejamento, tentativas de se matar e o próprio ato consumado (BAHIA CA, et al., 2020). Na epidemiologia do suicídio, variáveis têm implicações importantes, e, por isso, a importância de correlaciona-las e analisa-las.

O sexo feminino prevalece nas tentativas de suicídio no Brasil. Entretanto, é o sexo masculino que tem maior factibilidade no ato suicida de acordo com as notificações de atendimentos públicos de saúde (JUNIOR CJS, et al., 2019). Tal evidencia está em consonância com a pesquisa identificada na base de dados DATASUS.

Há explicações para esclarecer as causas das taxas elevadas de suicídio entre os homens, entre as quais, citam-se: a influência de variáveis socioeconômicas relacionadas à execução de papéis sociais que são atribuídos ao sexo masculino, o aproveitamento escolar e a capacidade cognitiva, além da resistência em procurar auxílio psiquiátrico, o efeito das relações afetivas mal sucedidas, bem como da alienação parental, a influência hormonal e a maior prevalência de alcoolismo (PALMA DCA, et al., 2020). Ademais, outros autores indagam que essa realidade pode se refletir por uma maior frequência de instrumentais letais, enquanto o sexo feminino utiliza meios de menor invasão letal (JUNIOR CJS, et al., 2019).

Em relação à faixa etária, a população jovem e adulta, entre 20 e 29 anos, possuiu o maior aumento nas taxas de violência auto infligida. Tal aumento foi relacionado em alguns estudos à situação socioeconômica, vivenciada no país desde meados do ano 2000 (RIBEIRO JM e MOREIRA MR; 2018). A crise econômica pode ter refletido diretamente em menores expectativas de crescimento e construção profissional a essa parcela em ascensão na população, o que caracteriza momentos de frustração e pode corroborar com o desenlace suicida (FILHO MC e ZERBINI T, 2016).

No que tange à variável raça, os resultados indicam maior número de lesões autoprovocadas entre brancos, assim como maior número de óbitos entre indivíduos dessa raça. Esses resultados corroboram os dados de estudos anteriores, no qual os autores traçaram o perfil epidemiológico de mortalidade por suicídio no Brasil entre 2006 a 2015 e encontraram maior número de suicídio na população branca, seguido da população parda (MATA KCR, et al., 2020). Entretanto, estudos realizados no Espírito Santo (TAVARES FL, et al.,2020) e no Ceará (PEDROSA NFC, et al., 2018), observaram maior prevalência de suicídio entre pardos. Portanto, é necessário cautela ao interpretar os dados obtidos em relação à raça, pois deve-se levar em consideração que os mesmos são provenientes da autodeclaração e podem estar enviesados, haja vista que a raça indicada pelo indivíduo pode diferir da sua verdadeira (MATA KCR, et al., 2020).

O conhecimento sobre suicídio nas Américas foi construído através da perspectiva dos interesses das elites colonizadoras brancas, as quais buscaram entender esse processo em seus termos, negando a humanidade aos não brancos (HERTZMAN MA, 2019). Diante disso, percebe-se a necessidade de estudos mais detalhados a respeito da variável raça, principalmente no Brasil, um país com bastante miscigenação, no qual a questão racial confunde-se com outros marcadores de desigualdade social, como a baixa escolaridade, o desemprego e a pobreza.

A respeito da escolaridade, observaram-se altos índices de casos ignorados ou em branco. A incompletude dos registros quanto à escolaridade prejudica os esforços para traçar o perfil do paciente que comete lesão autoprovocada e suicídio, refletindo diretamente nas políticas de prevenção que acabam não considerando essa variável. O preenchimento incompleto dos registros pode estar relacionado a fatores como a falta de conhecimento e treinamento dos profissionais de saúde com relação à importância do preenchimento correto da ficha de notificação para o desenvolvimento das políticas de saúde (SOUSA CMS, et al., 2020).

Observa-se que as taxas de mortalidade por lesão autoprovocada diminuem conforme os anos de escolaridade aumentam. Esses dados confirmam estudos anteriores que ressaltam a associação positiva entre baixa escolaridade e taxas de suicídio. A situação de pouco ou nenhum estudo, dificulta o acesso a 
empregos com melhor remuneração, prejudicando a renda familiar, fato que gera estresse e preocupação nos indivíduos. Além disso, o nível de escolaridade da pessoa afeta sua autoavaliação, interferindo em sua autoestima. Sabendo que a baixa autoestima, por sua vez, configura-se fator de risco para o suicídio (PEREIRA AS, et al.,2018).

As regiões brasileiras apresentaram diferentes tendências da mortalidade por suicídio. A região que apresentou maior aumento de casos no país foi à região Sudeste, seguida pela região Sul, Nordeste, CentroOeste e Norte. Observa-se que a região Sudeste lidera as internações por tentativa de suicídio, o que justifica maior procura e eficiência do socorro médico executado nos centros urbanos, além de questões relacionadas ao contexto regional/local, provavelmente melhores descrições/notificações em análises específicas por região/localidade (BAHIA CA, et al., 2020). É possível correlacionar mortalidade por suicídio no país com uma integração de aspectos psicológicos, biológicos, socioculturais e financeiros, justificando os elevados índices observados na região Sul, atingindo especialmente conglomerados familiares de agricultores expostos a pesticidas e que vivem mais isoladamente (BAHIA CA, et al., 2020).

Sobre o meio utilizado para lesões autoprovocadas intencionais, percebeu-se um sublime número de autointoxicação intencional por medicamentos e substâncias biológicas não especificadas (ROSA NM, et al., 2017). Outros estudos apontaram o envenenamento como meio mais frequente nas tentativas de suicídio entre o sexo feminino, ao passo que entre o sexo oposto o enforcamento foi o mais comum, o que fora também encontrado neste presente trabalho. Estes achados confirmam que o sexo masculino utiliza métodos mais irreversíveis e invasivos, resultando em taxas de óbitos mais proeminentes que as do sexo feminino (ROSA NM, et al., 2017).

No panorama atual, o número de óbitos por suicídio se mostrou crescente, demonstrando uma larga discrepância entre os anos de 1996 e 2019. Em virtude disso, se questiona a causalidade desse fenômeno; quer seja por uma tendência temporal de crescimento da mortalidade em si ou pela intensificação das medidas de notificação compulsória. Logo, é inegável o aumento dos casos de suicídio no Brasil, que por sua vez, ocorrem como consequência de um desfecho de uma série de fatores, também pautados na história individual de cada vítima. Ou seja, conjuntamente à consequência final de um processo multifatorial, como mencionado tanto pela Organização Mundial da Saúde quanto pela Associação Brasileira de Psiquiatria (KRUG EG, et al, 2002; FERREIRA FN e MOTTA ID, 2019).

Além disso, nos últimos anos, o Brasil vem buscando mecanismos a fim de propiciar a notificação compulsória do suicídio. A exemplo da Lei oㅜ 13.819/2019, que prevê em seu artigo 6ª inciso II e parágrafo $5^{\circ}$, a obrigatoriedade da notificação de casos suspeitos ou confirmados por estabelecimentos de ensino público e privado ao Conselho Tutelar; bem como por meio de orientação às instituições de educação, para que instruam seus profissionais a respeito dos procedimentos de notificação estabelecidos pela Política Nacional de Prevenção da Automutilação e do Suicídio (FERREIRA FNL e MOTTA ID, 2019). No entanto, a legislação é recente, não havendo como atestar ainda uma relação direta de sua eficácia.

A violência por repetição consiste na reincidência de tentativa de autoextermínio ou autoagressão, sendo que o fator de risco mais relevante para o suicídio é a tentativa anterior (PEREIRA AS, 2018). Estudos sobre o comportamento suicida revelaram fortes evidências de que o fato de uma pessoa haver tentado uma vez suicídio, aumenta muito a chance de ocorrer nova tentativa (MINISTÉRIO DA SAÚDE, 2020). Dessa forma, após uma tentativa de suicídio, dependendo da gravidade clínica investida, alguns pacientes necessitam atendimento em serviços de emergência (MINISTÉRIO DA SAÚDE, 2016). Contudo, na maioria das vezes não são encaminhadas para serviços especializados em saúde mental. Mas, com suas vulnerabilidades ainda expostas e sem o cuidado necessário, podem reincidir sua tentativa de autoextermínio.

Com relação à evolução dos casos, a maior parte dos dados não foram preenchidos nos registros, o que pode indicar que os atendimentos são pontuais, sem longitudinalidade no acompanhamento. Isto evidencia o despreparo dos profissionais de saúde para atender casos de autoagressão, uma vez que pessoas com histórico de autolesão constituem grupo de risco para o suicídio e devem ser acompanhadas visando prevenir a consumação do ato suicida (FONSECA PHN, et al., 2018). A continuidade do acompanhamento é necessária para um tratamento eficaz que ajude a pessoa no enfrentamento das situações que ocasionaram 
a crise suicida, com foco no ser humano de forma integral, não apenas nos aspectos fisiológicos. Nesse sentido, observa-se a incompetência do sistema de saúde no que se refere ao suicídio, isto se deve em parte ao preconceito e estigma relacionados ao tema (TAVARES FL, et al., 2020).

Além disso, outro dado importante encontrado nesse estudo foi o número considerável de mortes por suicídio ocorridas em estabelecimentos de saúde (aproximadamente $20 \%$ dos locais registrados). Essa estatística preocupante levanta diversos questionamentos: qual a eficácia do atendimento recebido por pessoas com tendências suicidas? Como elas estão colocando em prática seus planos suicidas em ambientes no quais deveriam estar sendo monitoradas para evitar tais ocorrências? Que mudanças poderiam ser implementadas para diminuir essas taxas? São perguntas que carecem de mais estudos direcionados a estes aspectos para serem respondidas.

Manter o paciente seguro é um dos principais objetivos do profissional ao atender um paciente suicida. A tentativa prévia aumenta o risco de consumação do suicídio, portanto algumas precauções devem ser tomadas no momento da internação. A equipe de saúde precisa ser notificada sobre o risco de suicídio, dedicando atenção redobrada em períodos de trocas de turnos. O cuidado direcionado ao paciente suicida precisa ir além dos procedimentos relacionados à saúde física, pois a equipe também necessita ter sensibilidade para lidar com os aspectos emocionais envolvidos na tentativa de suicídio. Os profissionais devem ter uma postura empática, buscando estabelecer vínculo com o paciente através da escuta atenta dos seus sentimentos, evitando julgamentos, pois o acolhimento tem potencial para acalmar o paciente e minimizar a intensidade dos seus sintomas (FONTÃO MC, et al., 2018).

\section{CONCLUSÃO}

Fatores sociais e biológicos são de risco para o suicídio com relação às variáveis sexo, aspectos geográficos, raça, escolaridade e idade. O sexo masculino apresenta maior taxa de mortalidade, enquanto a raça carece estudo sobre a realidade brasileira e regiões com sistema de saúde melhor apresentam mais notificações. Ademais, recentemente a crise econômica gerou incremento das taxas de suicídio na população com baixa escolaridade e nos jovens. Dessa forma, é essencial assistência adequada aos pacientes com tendências suicidas durante e pós-internação com capacitação dos profissionais da saúde de maneira sistemática além da notificação e preenchimento completo das fichas de óbito.

\section{REFERÊNCIAS}

1. BAHIA CA, et al. Notificações e internações por lesão autoprovocada em adolescentes no Brasil, 2007-2016. Epidemiologia e Serviços de Saúde, 2020; 29(2): 1-12.

2. BOSTWICK J, et al. Suicide Attempt as a Risk Factor for Completed Suicide: Even More Lethal Than We Knew. American Journal of Psychiatry, 2016; 173(11): 1094-1100.

3. BRASIL. LEI № 13.819, DE 26 DE ABRIL DE 2019. Institui a Política Nacional de Prevenção da Automutilação e do Suicídio, a ser implementada pela União, em cooperação com os Estados, o Distrito Federal e os Municípios; e altera a Lei no 9.656, de 3 de junho de 1998. Disponível em: http://www.planalto.gov.br/ccivil_03/_ato20192022/2019/Lei/L13819.htm. Acessado em: 22 de junho de 2021.

4. BUTTER S, et al. Negative self-evaluation and the genesis of internal threat: beyond a continuum of suicidal thought and behaviour. Psychological medicine, 2019; 49(15): 1-9.

5. CANO-MONTALBAN I, QUEVEDO-BLASCO R. Sociodemographic variables most associated with suicidal behaviour and suicide methods in Europe and America. A systematic review. The European Journal of Psychology Applied to Legal Context, 2018; e 1(10): 15-25.

6. FERREIRA FNL, MOTTA ID. O procedimento de notificação compulsória nos estabelecimentos de ensino na nova política nacional de prevenção da automutilação e do suicídio. Revista Jurídica, 2019; 1(54): 519-555.

7. FILHO MC, ZERBINI T. Epidemiologia do suicídio no Brasil entre os anos de 2000 e 2010 . Saúde, Ética \& Justiça, 2016; $21(2): 45-51$.

8. FONSECA PHN, et al. Autolesão sem intenção suicida entre adolescentes. Arquivos brasileiros de psicologia, 2018; 70(3): 246-258.

9. FONTÃO MC, et al. Nursing care to people admitted in emergency for attempted suicide. Revista Brasileira de Enfermagem, 2018; 71(5): 2199-2205. 
10. FRANKLIN JC, et al. Risk factors for suicidal thoughts and behaviors: A meta-analysis of 50 years of research. Psychol Bull, 2017; 143(2):187-232.

11. HERTZMAN MA. Diferenças fatais: suicídio, raça e trabalho forçado nas Américas. Revista Mundos do Trabalho, 2019; (11): 1-38.

12. JUNIOR CJS, et al. Perfil de pacientes atendidos por tentativa de suicídio em um hospital geral de Emergências do estado de Alagoas. Revista da USP, 2019; 52(3): 223-230.

13. MANN JJ, et al. Improving suicide prevention through evidence-based strategies: a systematic review. The American Journal of Psychiatry, 2021; 178(7): 611-624.

14. MARS B, et al. Predictors of future suicide attempt among adolescents with suicidal thoughts or non-suicidal self-harm: a population-based birth cohort study. Lancet Psychiatry, 2019; 6(4): 327-337.

15. MASI G, et al. Suicidal ideation, suicidal attempts and non-suicidal self-injuries in referred adolescent s. Riv Psichiatr. 2020; 55(3):168-174.

16. MATA KCR, et al. Perfil epidemiológico de mortalidade por suicídio no Brasil entre 2006 e 2015. Revista Psicologia, Diversidade e Saúde, 2020; 9(1): 74-87.

17. MINISTÁRIO DA SAÚDE. Fundação Oswaldo Cruz (FIOCRUZ). Saúde Mental e Atenção Psicossocial na Pandemia COVID-19, Suicídio na Pandemia COVID-19. Brasil, 2020. Disponível em: https://www.fiocruzbrasilia.fiocruz.br/wpcontent/uploads/2020/05/cartilha_prevencaosuicidio.pdf. Acessado em: 23 de julho de 2021.

18. MINISTÉRIO DA SAÚDE. Secretaria de Vigilância em Saúde, Departamento de Vigilância de Doenças e Agravos Não Transmissíveis e Promoção da Saúde. Viva: Instrutivo Notificação de Violência Interpessoal e Autoprovocada. 2.ed. 92 p.: il. $\quad 2016 . \quad$ Brasil, 2 Disponível https://bvsms.saude.gov.br/bvs/publicacoes/viva_instrutivo_violencia_interpessoal_autoprovocada_2ed.pdf. Acessado em: 13 de junho de 2021.

19. MINISTÉRIO DA SAÚDE. Secretaria Nacional da Família. Ministério da Mulher, da Família e dos Direitos Humanos Acolha a Vida: Porque a Vida Sempre Vale a Pena! Brasil, 2020. Disponível em: https://www.gov.br/mdh/ptbr/assuntos/acolha-a-vida/cartilha_orientcao-familias-v0809.pdf. Acessado em: 22 de junho de 2021.

20. ORGANIZAÇÃO MUNDIAL DE SAÚDE (OMS). Prevenção do suicídio: um manual para médicos clínicos gerais. 2019. Disponível em: https://www.gov.br/mdh/pt-br/assuntos/acolha-a-vida/bibliografia/omssuicideprev_gp_port.pdf/view. Acessado em: 11 de julho de 2021.

21. PALMA DCA, et al. Análise dos padrões espaciais e caracterização dos suicídios no Brasil entre 1990 e 2015. Caderno de Saúde Pública, 2020; 36(4): 1-13.

22. PEDROSA NFC, et al. Análise dos principais fatores epidemiológicos relacionados ao suicídio em uma cidade no interior do Ceará, Brasil. Revista de Saúde e Ciências Biológicas, 2018; 6(4): 399-404.

23. PEREIRA AS, et al, Fatores de risco e proteção para tentativa de suicídio na adultez emergente, Ciência \& Saúde Coletiva, 2018; 23(11): 3767-3777.

24. PREFEITURA DA CIDADE DO RIO DE JANEIRO. Secretaria Municipal de Saúde (SMS). Coleção Guia de Referência Rápida, Avaliação do Risco de Suicídio e sua Prevenção. 1aㅡ edição. Rio de Janeiro, 2016. Disponível em: https://subpav.org/download/prot/Guia_Suicidio.pdf. Acessado em: 22 de junho 2021.

25. RIBEIRO JM, MOREIRA MR. Uma abordagem sobre o suicídio de adolescentes e jovens no Brasil. Ciência \& Saúde Coletiva, 2018; 23(9): 2821-2834.

26. RIBEIRO NM, et al. Análise da tendência temporal do suicídio e de sistemas de informações em saúde em relação às tentativas de suicídio. Texto e Contexto - Enfermagem, 2018; 27(2): 1-11.

27. ROBINSON K, et al. Nonsuicidal self-injury thoughts and behavioural characteristics: Associations with suicidal thoughts and behaviours among community adolescents, Journal of Affective Disorders, 2021; (282): 1247-1254.

28. ROSA NM, et al. Mortalidade por suicídio no Estado do Paraná segundo meios utilizados: uma análise epidemiológica. Revista Brasileira de Psiquiatria, 2017; 66(2): 73-82.

29. SOUSA CMS, et al. Incompletude do preenchimento das notificações compulsórias de violência - Brasil, $2011-2014$. Cadernos Saúde Coletiva, 2020; 28(4): 477-487.

30. TAVARES FL, et al. Mortalidade por suicídio no Espírito Santo: uma análise do período de 2012 a 2016 . Avances em Enfermagem, 2020; 38(1): 66-76.

31. TAYLOR PJ, et al. A meta-analysis of the prevalence of different functions of non-suicidal self-injury. J Affect Disord, 2018; (227): 759-769.

32. WITT K, et al. Population attributable risk of factors associated with the repetition of self-harm behaviour in young people presenting to clinical services: a systematic review and meta-analysis. Eur Child Adolesc Psychiatry, 2019; 28(1): 5-18. 